\title{
A CONVOLUTION EQUATION AND HITTING PROBABILITIES OF SINGLE POINTS FOR PROCESSES WITH STATIONARY INDEPENDENT INCREMENTS
}

\author{
BY HARRY KESTEN 1
}

Communicated by Henry P. McKean, Jr., November 19, 1968

In this note we study the hitting probability of a point $r$,

$$
h(r)=P\left\{X_{t}=r \text { or } X_{t-}=r \text { for some } 0<t<\infty\right\},
$$

for a $d$-dimensional right continuous process $\left\{X_{t}\right\}_{t \geq 0}$ with stationary independent increments and $X_{0}=0$ with probability 1 . It is well known (see $[7, \S 62],[1, \S 3.4]$, or $[5, \S 4]$ ) that the characteristic function for any such process is of the form

(1) $E e^{i \lambda \cdot x_{t}}=\exp \left\{i \lambda \cdot a t-\frac{1}{2} Q(\lambda) t+t \int\left[e^{i \lambda \cdot y}-1-\frac{i \lambda \cdot y}{1+|y|^{2}}\right] \nu(d y)\right\}$, $\lambda=\left(\lambda_{1}, \cdots, \lambda_{d}\right) \in R^{d}, a \in R^{d}, Q$ a nonnegative definite quadratic form and $\nu$ a Borel measure on $R^{d}-\{0\}$ for which

$$
\int \min \left(1,|y|^{2}\right) \nu(d y)<\infty \text {. }
$$

Our purpose is to determine when $h(r)$ is strictly positive, respectively zero. An old and obvious result is that $h(r)>0$ for all $r$ if $X_{t}$ is Brownian motion. Somewhat more difficult is the behavior of $h(r)$ for symmetric stable processes of index $\alpha$ in dimension one. For such processes it was shown by Lévy, Erdös and Kac (see [6]; also [12] for simplified proofs) that $h(r)>0$ for all $r \in R$ if $1<\alpha \leqq 2$ and $h(r)=0$ for all $r \in R$ if $\alpha \leqq 1$. It will be seen that this result is typical for the general situation as well. The motivation for our study was a problem of Chung's [2], [3], which asked to solve the one-dimensional convolution equation

$$
\int_{0-}^{r+} \sigma(r-s) W(d s)=1, \quad r>0 .
$$

Here $\sigma$ is a given decreasing right continuous function on $[0, \infty)$ such

${ }^{1}$ Research supported by the NSF under grant GP 7128. 
that $^{2}$

$$
\sigma(y) \downarrow 0 \quad \text { as } y \rightarrow \infty \text { and }\left|\int_{0}^{\infty} \min (1, y) d \sigma(y)\right|<\infty \text {, }
$$

whereas one looks for a Borel measure $W$ which satisfies (2). Using Laplace transforms, Neveu [11] and Chung [2], [3] found what the only possible solution of (2) could be. Let $\left\{X_{t}\right\}_{t \geq 0}$ be a 1-dimensional right continuous process with stationary independent increments with characteristic function

$$
E e^{i \lambda x_{t}}=\exp \left\{-t \int_{0}^{\infty}\left(e^{i \lambda y}-1\right) d \sigma(y)\right\}
$$

and put $^{3}$

$$
\begin{aligned}
W(A) & =E \int_{0}^{\infty} I_{\Lambda}\left(X_{t}\right) d t=\int_{0}^{\infty} P\left\{X_{t} \in A\right\} d t \\
& =\text { expected amount of time spent in } A \text { by }\left\{X_{t}\right\} .
\end{aligned}
$$

For this choice of $W(2)$ is satisfied for (Lebesgue) almost all $r>0$ and no other Borel measure $W$ will satisfy (2) for almost all $r>0$. Chung [2] raised the problem whether (5) solves (2) for all $r>0$ and showed that this is indeed the case if $\sigma(0)<\infty$ or if $\sigma$ satisfies certain smoothness assumptions ([2], [3]; see also $[10, \S I V])$. Neveu $[11$, lemma on p. 40] already stated that (2) holds for all $r>0$ with $W$ as in (5), but his proof is not convincing (see footnote 2 in [3]) and another recent proof $[8]$, also contains a gap. (See $[10$, just before the second proposition in \$IV] as well as [9].)

Our attention was drawn to this problem by P. A. Meyer ${ }^{4}$ when he pointed out to us that in case $\sigma(0)=\infty$, one has (with $W$ given by (5)) ${ }^{5}$

$$
\begin{aligned}
& \int_{0-}^{r+} \sigma(r-s) W(d s) \\
& \quad=1-P\left\{X_{t}=r \text { or } X_{t-}=r \text { for some } 0<t<\infty\right\},
\end{aligned}
$$

2 We allow $\sigma(0)=\sigma(0+)=\infty$; but $0 \leqq \sigma(y)<\infty$ for all $y>0$ by (3).

${ }^{3} I_{A}(\cdot)$ is the characteristic function of $A$.

- We wish to thank Professor Meyer for introducing us to this nice problem and especially for pointing out the purely probabilistic nature of the problem as exhibited by (6). It was this observation which led us to study the hitting probability $h(r)$.

- (6) remains valid even if $X_{t}$ has a positive drift, i.e., if (4) is generalized to $E e^{i \lambda x_{i}}=\exp \left\{i a t-t \int_{0}^{\infty}\left(e^{i \lambda y}-1\right) d \sigma(y)\right.$ with $a \geqq 0$. 
(see \&IV of [10]; another proof of (6) is contained in Theorem 1 of [4]). This reduces Chung's problem in the case $\sigma(0)=\infty$ to showing $h(r) \equiv 0$ for the $X$ process. This fact is indeed contained in part (c) of Theorem 1 below. Together with Chung's result for $\sigma(0)<\infty$ (see also §IV of [10]) this answers Chung's question affirmatively. Theorems 1-3 pretty much determine when $h(r)>0$, resp. $h(r)=0$. To state the theorems for $d=1$ we make one more convention. If $d=1$ we shall always write $Q(\lambda)$ as $\lambda^{2} \sigma^{2}$ and if

$$
\int_{-2}^{+1}|y| \nu(d y)<\infty
$$

then we write

$$
\tilde{a}=\int_{-\infty}^{+\infty} \frac{y}{1+|y|^{2}} \nu(d y)
$$

and express the characteristic function of $X_{t}$ as

$$
E e^{i \lambda x_{t}}=\exp \left\{i a^{\prime} t-\frac{\lambda^{2} \sigma^{2}}{2}+t \int_{-\infty}^{+\infty}\left(e^{i \lambda y}-1\right) \nu(d y)\right\}
$$

with $a^{\prime}=a-\tilde{a}$. In particular the representation (8) will be used if $\nu(R-\{0\})<\infty$.

Theorem 1. Let $\left\{X_{t}\right\}_{t \geq 0}$ be a 1-dimensional right continuous process with stationary independent increments and characteristic function (1) with $\lambda^{2} \sigma^{2}$ written for $Q(\lambda)$. Then exactly the following possibilities for $h(\cdot)$ exist:

(a) If $\sigma^{2}>0$ then $h(r)>0$ for all $r$.

(b) If $\sigma^{2}=0, \nu(R-\{0\})<\infty$ and $a^{\prime}=0$ then $h(r)>0$ if and only if $r=0$ or $r \in \Xi=$ the semigroup generated by the atoms of $\nu$.

(c) If $\sigma^{2}=0, \nu(R-\{0\})=\infty$, but $\int_{-1}^{+1}|y| \nu(d y)<\infty$ and $a^{\prime}=0$ then $h(r)=0$ for all $r$.

(d) If $\sigma^{2}=0, \int_{-1}^{+1}|y| \nu(d y)<\infty$ and $a^{\prime}>0$ together with $\nu((-\infty, 0))$ $>0$ or $a^{\prime}<0$ together with $\nu((0, \infty))>0$ then $h(r)>0$ for all $r$.

(e) If $\sigma^{2}=0, \int_{-1}^{+1}|y| \nu(d y)<\infty$ and $a^{\prime}>0$ but $\nu((-\infty, 0))=0$ then $h(r)>0$ if and only if $r>0$. If $\sigma^{2}=0, \int_{-1}^{+1}|y| \nu(d y)<\infty$ and $a^{\prime}<0$ but $\nu((0, \infty))=0$, then $h(r)>0$ if and only if $r<0$.

(f) If $\sigma^{2}=0$ and

$$
\int_{0}^{1} y \nu(d y)=\infty \text { together with } \int_{-1}^{0}|y| \nu(d y)<\infty
$$


or

$$
\int_{0}^{1} y \nu(d y)<\infty \text { together with } \int_{-1}^{0}|y| \nu(d y)=\infty,
$$

then $h(r)>0$ for all $r$.

(g) If $\sigma^{2}=0$ and

$$
\int_{0}^{1} y \nu(d y)=\int_{-1}^{0}|y| \nu(d y)=\infty,
$$

then either $h(r)>0$ for all $r$ or $h(r)=0$ for all $r$.

In case (g) of Theorem 1 both situations can arise. E.g. by the results in [6] and [12] quoted above, $h(r) \equiv 0$ for the symmetric Cauchy process and $h(r)>0$ for the symmetric stable processes of index $\alpha>1$. The next theorem gives a criterion to decide which case occurs. In most practical situations one will have a hard time applying the criterion, though.

ThEOREM 2. Under the assumptions of Theorem $1, h(r)=0$ for almost all $r \in R$ if and only if

$$
\begin{aligned}
\int_{-\infty}^{+\infty}\left\{\gamma+\frac{\lambda^{2} \sigma^{2}}{2}\right. & \left.+\int_{-\infty}^{+\infty}(1-\cos \lambda y) \nu(d y)\right\} \\
\left\{\left[\gamma+\frac{\lambda^{2} \sigma^{2}}{2}\right.\right. & \left.+\int_{-\infty}^{+\infty}(1-\cos \lambda y) \nu(d y)\right]^{2} \\
& \left.+\left[a \lambda+\int_{-\infty}^{+\infty}\left(\sin \lambda y-\frac{\lambda y}{1+y^{2}}\right) \nu(d y)\right]^{2}\right\}^{-1} d \lambda=\infty
\end{aligned}
$$

for each $\gamma>0$.

If $\nu(R-\{0\})=\infty$ we may replace " $h(r)=0$ for almost all $r \in R$ " by ${ }^{\alpha} h(r)=0$ for all $r \in R . "$

For $d \geqq 2$ the full list of possibilities for $h(r)$ is even larger than in Theorem 1. We therefore only give a general statement valid for "honestly $d$-dimensional" processes, $d \geqq 2$.

TheOREM 3. Let $\left\{X_{t}\right\}_{\text {tzo }}$ be a d-dimensional, right continuous process with stationary independent increments and characteristic function (1) with $d \geqq 2$. If $\nu\left(R^{d}-F\right)=\infty$ for any $(d-1)$-dimensional linear subspace $F$ then $h(r)=0$ for all $r$. Also $h(r)=0$ for all $r$ when $X$, has an absolutely continuous distribution for all $t>0$. This last case occurs if $Q(\cdot)$ is strictly positive definite. 
The detailed proofs will appear elsewhere but we indicate a few steps here. First we observe that

$$
h(r)=P\left\{X_{t}=r \text { for some } 0<t<\infty\right\}
$$

by quasi-left-continuity of the $X_{t}$ process, and then, by the strong Markov property

$$
h(r+s) \geqq h(r) h(s)
$$

(see also §IV of [10]). The easy cases (a), (d) and (f) of Theorem 1 are then proved by showing

$$
\int h(r) d r=E\left(\text { Lebesgue measure of range of }\left\{X_{\ell}\right\}\right)>0 .
$$

In essence one shows in these cases that $X_{1}$ has bigger fluctuations than can be caused by the jumps alone so that $\operatorname{cl}\left\{X_{t}: t \geqq 0\right\}$, the closed range of the process, has positive Lebesgue measure with probability 1 . Once $h(r)>0$ on a set of positive measure (as implied by (10)) one derives $h(r)>0$ everywhere from (9). Case (e) of Theorem 1 follows from (6) (with footnote 5), the lemma on p. 40 of [11] and (9) again. The really difficult parts are case (c) of Theorem 1 , case (g) of Theorem 1 if $h(r)$ is not everywhere positive, and Theorem 3 without continuity assumptions for the distribution of $X_{t}$. For these cases we first show that $h(r)=0$ a.e. We then put

$$
S_{r}=\inf \left\{t>0: X_{t}=r\right\}
$$

and use the following consequence of the strong Markov property:

$$
\begin{aligned}
U(r-\epsilon, r+\epsilon) & =\int_{0}^{\infty} e^{-\gamma t} P\left\{r-\epsilon \leqq X_{t} \leqq r+\epsilon\right\} d t \\
& \geqq E e^{-\gamma S_{r}} \int_{0}^{\infty} e^{-\gamma t} P\left\{-\epsilon \leqq X_{t} \leqq+\epsilon\right\} d t \\
& =U(-\epsilon,+\epsilon) E e^{-\gamma S_{r}} .
\end{aligned}
$$

Lastly, one shows by a complicated renewal argument that for fixed $r>0, r \neq 0$

$$
\liminf _{|\epsilon| t_{0}} \frac{U(r-\epsilon, r+\epsilon)}{U(-\epsilon,+\epsilon)}=0,
$$

whence $E e^{-\gamma s r}=0$ and $h(r)=0$ (at least for $r \neq 0$ ).

- For $d \geqq 2, a \leqq X_{t} \leqq b$ is used as an abbreviation for the simultaneous inequalities $a_{i} \leqq X_{t}^{(i)} \leqq b_{i}, i=1, \cdots, d$, on the components $X_{t}^{(i)}$ of $X_{t}$. 


\section{REFERENCES}

1. S. Bochner, Harmonic analysis and the theory of probability, Univ. of California Press, Berkeley, Calif., 1955.

2. K. L. Chung, Sur une equation de convolution, C. R. Acad. Sci. Paris 260 (1965), 4665-4667.

3. - Sur une equation de convolution, C. R. Acad. Sci. Paris 260 (1965), 6794-6796.

4. N. Ikeda and S. Watanabe, On some relations between the harmonic measure and the Levy measure for a certain class of Markov processes, J. Math. Kyoto Univ. 2 (1962), 79-95.

5. K. Ito, Lectures on stochastic processes, Tata Institute of Fundamental Research, Bombay, 1961.

6. M. Kac, Some remarks on stable processes, Publ. Inst. Statist. Univ. Paris 6 (1957), 303-306.

7. P. Lévy, Thérie de l'addition des variables aleatoires, 2nd ed., Gauthier-Villars, Paris, 1954.

8. H. P. McKean, Jr., An integral equation arising in connection with Markov chains, Z. Wahrscheinlichkeitstheorie und Verw. Gebiete 8 (1967), 298-300.

9. - Correction to: An integral equation arising from Markov chains, Z. Wahrscheinlichkeitstheorie und Verw. Gebiete 11 (1968), 82.

10. P. A. Meyer, Processus d accroissements indépendants et positifs, Séminaire de Probabilités III, Université de Strasbourg, Springer Lecture Notes in Math. (to appear).

11. J. Neveu, Une géneralisation des processus a accroissements positifs indépendants, Abh. Math. Sem. Univ. Hamburg 25 (1961), 36-61.

12. S. C. Port, Hitting times and potentials for recurrent stable processes, J. Analyse Math. 20 (1967), 371-395.

Aarhus University, Aarhus, Denmark aND

Cornell University, Ithaca, New York 14850 